\title{
Model Reference PI Controller Tuning for Second Order Inverse Response and Dead Time Processes
}

\author{
J.A. Martinez ${ }^{\dagger}$, O. Arrieta ${ }^{\dagger}$, R. Vilanova ${ }^{\star}$, J.D. Rojas ${ }^{\dagger}$, L. Marin ${ }^{\dagger}$, M. Barbu ${ }^{\star}, \S$ \\ ${ }^{\dagger}$ Instituto de Investigaciones en Ingeniería, Facultad de Ingeniería, \\ Universidad de Costa Rica, 11501-2060 San José, Costa Rica. \\ ${ }^{\star}$ Departament de Telecomunicació i d'Enginyeria de Sistemes \\ Universitat Autònoma de Barcelona, 08193 Bellaterra, Barcelona, Spain. \\ $\S$ Department of Automatic Control and Electrical Engineering, \\ "Dunarea de Jos" University of Galati, 800008 Galati, Romania.
}

\begin{abstract}
In this paper, a One-Degree-of-Freedom PI controller is optimized using the model reference tuning approach for a Second Order Inverse Response and Dead Time Process operating as a servo control. In addition, a graphic user interface tool that computes the PI optimized controller parameters is presented, also showing the response of the control system operating as a servo-control (the optimized one) and the associated response for the regulatory-control case.
\end{abstract}

\section{INTRODUCTION}

Nowadays the most used and implemented feedback controller in the industry is the PID (Proportional - Integral Derivative) controller, as it provides a satisfactory performance in most cases [1]. The design of these type of controllers is usually done to obtain a good disturbance rejection, as the setpoint in most industrial applications is not modified frequently.

When designing the controller two main approaches can be followed, designing the controller when the set-point tracking is the main objective is known as servo-control, and when a good disturbance rejection is needed, the controller can be tuned as a regulatory-control [2], [3]. To guarantee a stable and successful system operation, the controller must be tuned according to these operation modes and according to controlled process [1]. For this objective, several tuning methods exist in the literature, providing the optimal PID controller parameters according to the industrial process [4].

Although in industrial processes the emphasis needed in most cases is the regulatory-control, neglecting the set-point tracking can be counterproductive in some cases. In this case, when a good performance is needed in both operating modes, a well-known problem occurs, as there is a compromise between the performance of a servo and a regulatory control as the usual one degree of freedom (1DoF) PID controller can only be optimized to one mode of operation, and this will degrade the performance of the other operating mode [5]-[7]. To solve this issue, a Two-Degrees-of-Freedom

O. Arrieta email: Orlando.Arrieta@ucr.ac.cr 978-1-5090-1314-2/16/\$31.00 (C)2016 IEEE
(2DoF) PID controller can be implemented, as it includes a set-point weight factor $\beta$ that can improve the servo-control performance when the controller is designed first to optimize the regulatory-control behavior.

In order to obtain the PID parameters (tuning), currently there are many techniques in the literature [8]. Most of them take into account a design parameter, such as the settling time, the percent overshoot, the rising time and other performances indicators such as the integral indexes. But other aspects of the control-loop performance such as the system robustness, the control output evolution or the controller fragility must be also taken into account when tuning the controller, in order to guarantee an adequate performance of the control-loop in many circumstances [1]. When these specifications have to be achieved simultaneously, it can become a challenging issue in both $1 \mathrm{DoF}$ and $2 \mathrm{DoF}$ PID controllers. In this case, optimization techniques have to be used in order to find the PID parameters that fulfill two or more specifications simultaneously and, in some cases, when the control-loop have to operate in both servo and regulatory control.

In this paper, a One-Degree-of-Freedom (1DoF) PI controller is optimized for servo-control, taking into account the robustness of the control-loop defined with the maximum sensibility function $M_{s}$ and the performance of the system, defined via a similar model reference approach proposed by [9]. The novelty of this proposed approach lies in the extension of the current Model Reference Robust Tuning (MoReRT) [10] to a Second Order Inverse Response and Dead Time Process (previous one was without dead-time). Even the addition of the parameter for the delay can appear simple, it gives a lot of more information about the optimal points that is not trivial to manage. This work is a preliminary stage that must be continued with the tuning of 1DoF PI controller for regulatory-control and 2DoF PI controllers.

The rest of the paper is organized as follows: the control system framework is presented in Section II; the controller design methodology is described in Section III; 


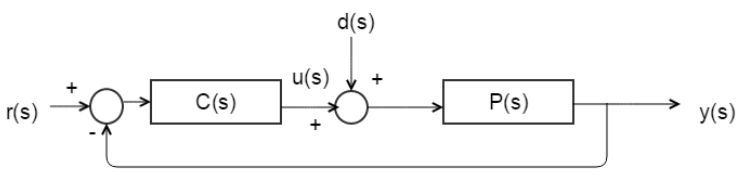

Figure 1. Closed-loop control system

the optimization results and the robustness obtained for the inverse response model, along with the developed interface are shown in Section IV; and finally some conclusions and future work are outlined in Section V.

\section{Problem Formulation}

The structure of an ideal PID (Proportional-IntegralDerivative) controller has three parameters that are the integral time $T_{i}$, the derivative time $T_{d}$ and the controller gain $K_{c}$.

In the present work the PI controller is utilized, thus $T_{d}$ is zero. The output expression for the $1 \mathrm{DoF}$ PI controller is defined through equation (1) in the time domain, being $e(t)$ the error signal.

$$
u(t)=K_{c}\left\{e(t)+\frac{1}{T_{i}} \int_{0}^{t} e(\tau) d \tau\right\},
$$

One of the advantages of using a PI controller is the elimination of the steady-state error. The controller equation in the frequency domain is obtained by taking the Laplace Transform of (1) as shown in (2).

$$
u(s)=K_{c}\left\{1+\frac{1}{T_{i} s}\right\} e(s),
$$

The closed-loop control system is shown in Fig. 1. In this, $P(s)$ is the controlled process, assumed as a second order inverse response and dead time model and $C(s)$ is the $1 \mathrm{DoF}$ PI controller. In this system, $r(s)$ is the set-point, $u(s)$ the controller output signal, $d(s)$ the load-disturbance and $y(s)$ the process controlled variable (system output).

The output of the closed loop system $y(s)$ is given by

$$
y(s)=M_{y r}(s) r(s)+M_{y d}(s) d(s)=y_{r}(s)+y_{d}(s)
$$

where $M_{y r}(s)$ and $M_{y d}(s)$ are the servo-control (set-point tracking) and regulatory-control (disturbance rejection) closedloop transfer functions, that are given by

$$
M_{y r}(s)=\frac{C(s) P(s)}{1+C(s) P(s)}
$$

$$
M_{y d}(s)=\frac{P(s)}{1+C(s) P(s)}
$$

In the formulation of the model reference robust approach, a reference model has to be stated, in order to use it in the optimization [9]. First it is necessary to define the model for the process that in this case is a Second Order Inverse Response and Dead Time shown in (6), where $K$ is the gain, $T$ the main time constant, $a$ the ratio of the two main poles time constants $(0.1 \leq a \leq 1.0), b$ the relative position of the right-half plane zero and $L$ the dead-time. The parameter set of this model is denoted by $\bar{\theta}_{p}=\{K, T, a, b, L\}$.

$$
P(s)=\frac{K(-b T s+1) e^{-L s}}{(T s+1)(a T s+1)},
$$

The reference model required for the process model (6) is the result of the modification performed to the equations proposed in [10] by adding the corresponding dead-time, having as result the target transfer function shown in (7) for the desired control system response to a set-point change, and in (8) for the desired control system response to a load-disturbance change.

$$
\begin{gathered}
y_{r}^{t}(s)=\frac{(-b T s+1) e^{-L s} s}{\left(\tau_{c} T s+1\right)\left(a \tau_{c} T s+1\right)} r(s), \\
y_{d}^{t}(s)=\frac{\left(T_{i} / K_{c}\right)(-b T s+1) e^{-L s} s}{\left(\tau_{c} T s+1\right)^{2}\left(a \tau_{c} T s+1\right)} d(s),
\end{gathered}
$$

In (7) and (8), the dimensionless design parameter $\tau_{c}$ represents the relative velocity time constant of closed-loop system. The global control system output target $y^{t}$ can be defined as

$$
y^{t}(s)=M_{y r}(s) r(s)+M_{y d}(s) d(s)=y_{r}^{t}(s)+y_{d}^{t}(s),
$$

A comparison of closed-loop system output (3) with desired output of the reference model (9) is performed via optimization, to guarantee that the difference between them is minimum. In this case the optimization will provide the optimal normalized PI controller parameters, as described in the next section.

\section{Controller Design And Tuning}

As was discussed on Section II, the general reference model is described by (9), however, as this paper focuses only (first stage) on the servo-control operation, only (7) will be used in the optimization, that will compare (7) to the response (4) (in the time domain), by means of a Cost Functional that computes the difference between the responses of both models.

According to the work presented in [10], the expression that represents the cost functional for the servo-control as 


$$
J_{r}=\int_{0}^{\infty}\left[y_{r}^{t}\left(\tau_{c}, \bar{\theta}_{p}, t\right)-y_{r}\left(\bar{\theta}_{c}, \bar{\theta}_{p}, t\right)\right]^{2} d t
$$

where $y_{r}^{t}$ is the step response of the servo-control closed-loop target transfer function, and $y_{r}$ is the step response of the servo-control closed-loop transfer function. By optimizing (11) the optimal PI controller parameters $\bar{\theta}_{c}=\left\{K_{c}, T_{i}\right\}$ are obtained.

$$
J_{T}^{o}=\min _{\bar{\theta}_{c}}\left\{J_{r}\left(\tau_{c}, \bar{\theta}_{c}, \bar{\theta}_{p}\right)\right\},
$$

The control system robustness $M_{s}$ is defined with the sensibility function on (12).

$$
M_{s}=\max _{\omega}|S(j \omega)|=\max _{\omega} \frac{1}{|1+C(j \omega) P(j \omega)|},
$$

The design looks to accomplish the robustness levels defined at the values of $\left.M_{s} \in\{1.4,1.6,1.8,2.0\}\right]$.

The optimal parameters can be achieving performing the optimization problem using MATLAB $®$.

The comparison between the real a reference model has to be close to zero, but that is just an ideal case, so this value has a tolerance of $1 \times 10^{-6}$, so the process will optimized the controllers parameters until the cost functional is $1 \times 10^{-6}$ or less.

The resulting parameters from the optimization are $\kappa_{p}$ (the normalized controller gain) and $\tau_{i}$ (the normalized controller integral time). The results of the optimizations of these parameters have been collected for a specific case and showing in the Figs. 2 and 3.

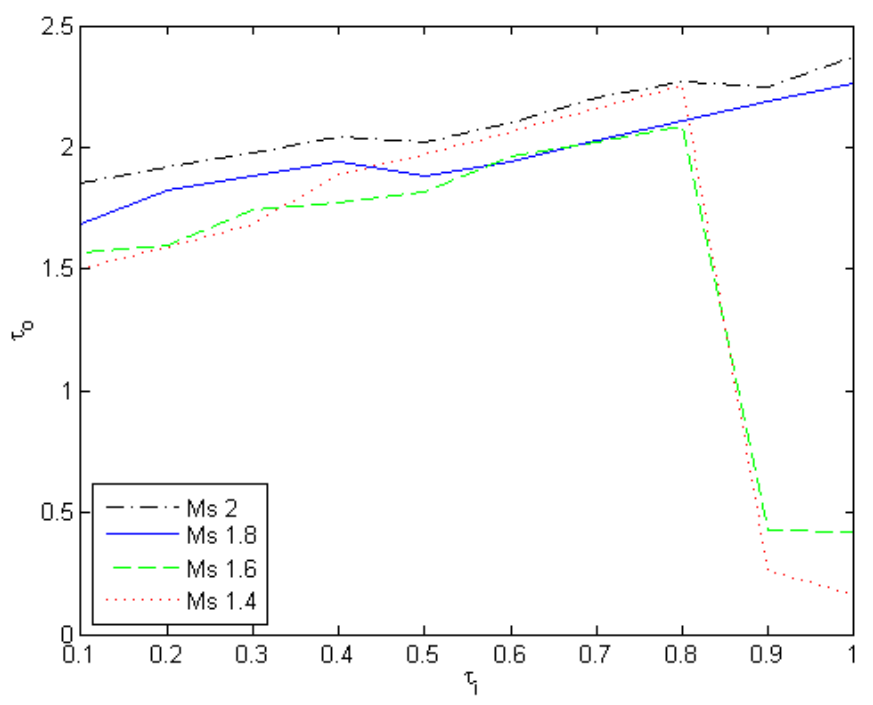

Figure 2. Normalized Integral Time with $a=0.5$ y $b=2$

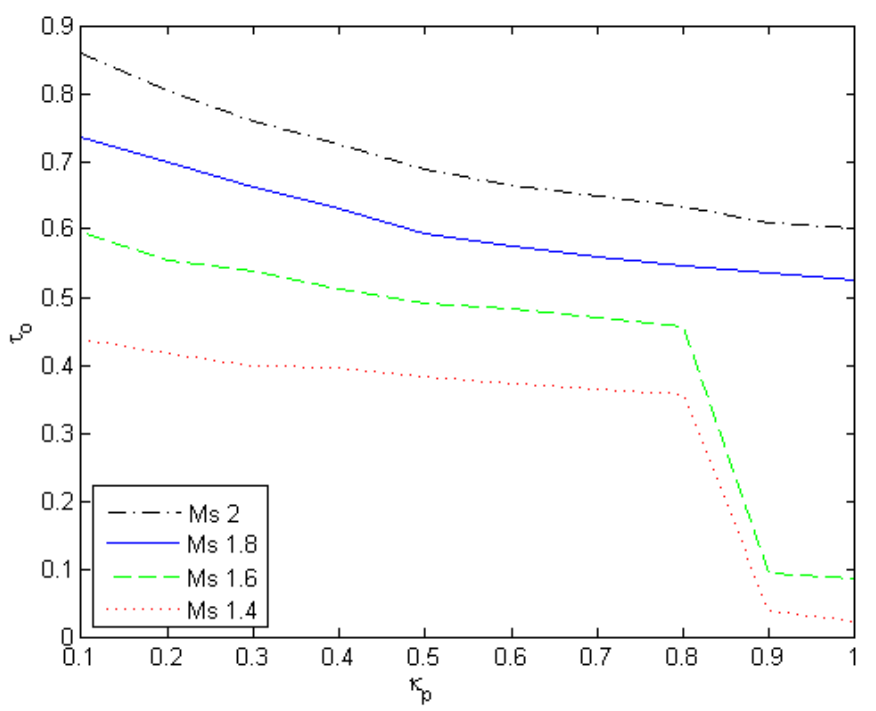

Figure 3. Controlled process normalized gain con $a=0.5$ y $b=2$

\section{Optimization Results and DeVEloped Interface}

In Figs. 2 and 3 are presented the variation of the controller parameters, from the results provided by the optimization procedure, for the all cases of robustness values.

In addition, taking into account that one of the goals of this approach is to satisfy a certain robustness level (from the value of $M_{s}$ ), in Figs. 4, 5 and 6 are shown some cases of the resulting values that can be achieved with the proposal, being these very close to the selected ones as desired values. Therefore, the accomplishment of the robustness for the control system is good enough.

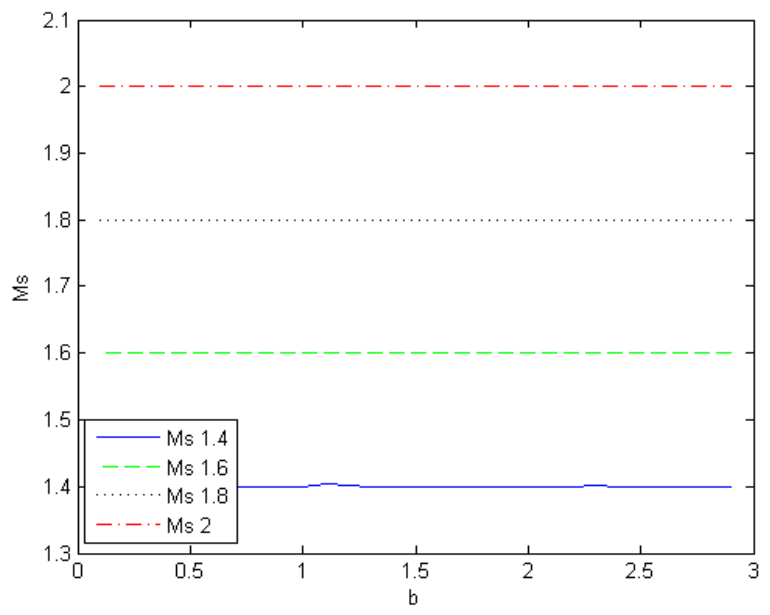

Figure 4. Accomplishment for the target robustness for the control system with $a=0.5$

The corresponding closed-loop responses to a step change in the set-point for the model (6) with $K=2.0, T=1.0$, 


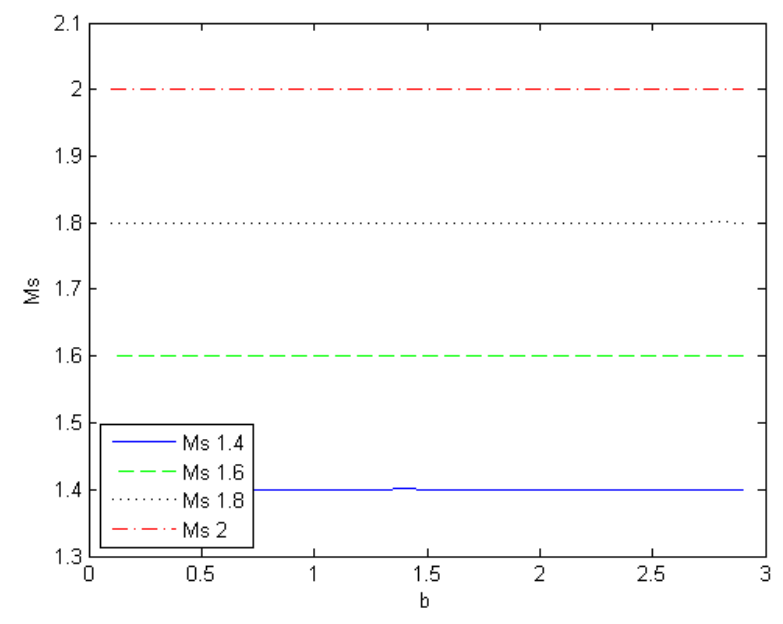

Figure 5. Accomplishment for the target robustness for the control system with $a=0.7$

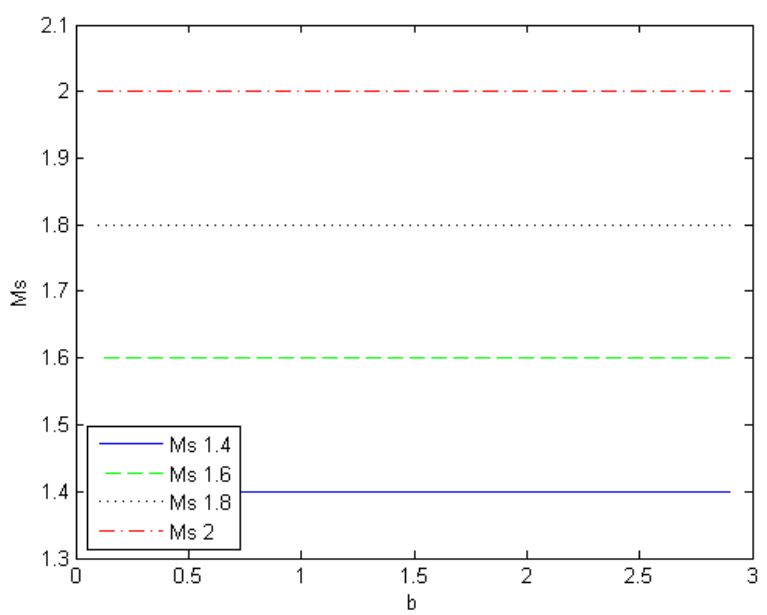

Figure 6. Accomplishment for the target robustness for the control system with $a=1.0$

$a=0.5, b=1.3$ and $L=0.6$, are shown in Fig. 7, for servo control operation.

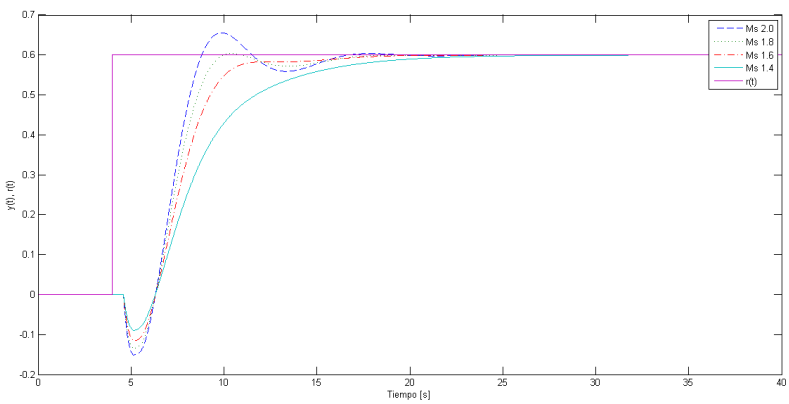

Figure 7. Control System Responses in Servo-Control
Taking into account that to achieve the PI controller parameters, it is necessary to variate the five parameters the model (6), the generated information of the optimal point must be huge and difficult to fit in a tuning equation rules. Therefore, it was developed a graphic interface tool in order to easy compute the controller parameters.

Fig. 8 shows the main screen of the interface, where the user should enter the information of the model, to achieve the PI parameters.

As an example in Fig. 9 there is entered a model with $K=3.0, T=5.0, a=0.5, b=1.2$ and $L=2.0$, computing the controller parameters as $K_{c}=0.116$ and $T_{i}=6.779$. Also in Figs. 10 and 11 are presented the control system responses for servo-control and regulatory-control operation, respectively.

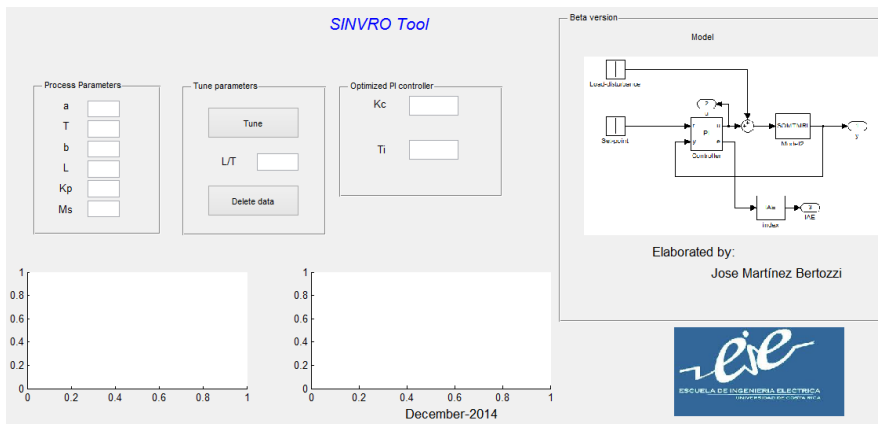

Figure 8. Main screen of the graphic interface tool

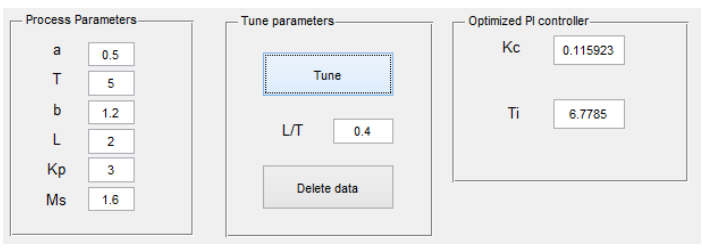

Figure 9. Practical example of the use of the interface tool

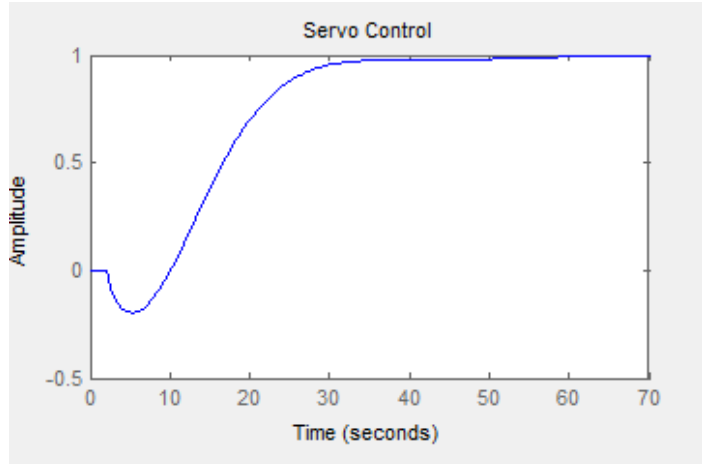

Figure 10. Servo-control response 


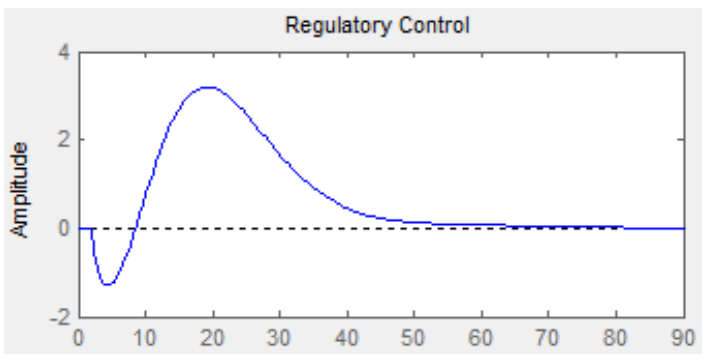

Figure 11. Regulatory-control response

\section{CONCLUSIONS AND Future WORK}

As an extension of the model reference tuning presented in [9], [10], it was proposed a design for second order inverse response models with also a dead-time, to achieve the $1 \mathrm{DoF}$ PI controller parameters for servo-control operation. The tuning guarantees the accomplishment of a certain desired robustness value into the option $M_{s} \in\{1.4,1.6,1.8,2.0\}$.

Taking into account the amount of information of the optimal tuning points, resulting from the optimization procedure, a graphical user interface tool was developed with the aim to facilitate the computation of the controller parameters.

This study is just a stage of a more general work, that is in progress, and must include the develop of designs for also 1 DoF PI controller in regulatory-control mode and for $2 \mathrm{DoF}$ PI controllers. Then it could be looking for tunings for PID controllers. In addition, the graphical interface tool should be improved including more quantitative measures for the performance and robustness of the control system.

\section{ACKNOWLEDGMENTS}

The financial support from the University of Costa Rica, under the grants 731-B4-213 and 322-B4-218, is greatly appreciated. Also, this work has received financial support from the Spanish CICYT program under grant DPI2013-47825-C3$1-\mathrm{R}$.

\section{REFERENCES}

[1] K. Åström and T. Hägglund, Advanced PID Control. ISA - The Instrumentation, Systems, and Automation Society, 2006.

[2] O. Arrieta and R. Vilanova, "PID Autotuning settings for balanced Servo/Regulation operation," in 15th IEEE Mediterranean Conference on Control and Automation (MED07), June 27-29, Athens-Greece, 2007.

[3] — , "Performance degradation analysis of controller tuning modes: Application to an optimal PID tuning," International Journal of Innovative Computing, Information and Control, vol. 6, no. 10, pp. 4719-4729, 2010.

[4] V. M. Alfaro, R. Vilanova, and O. Arrieta, "A Single-Parameter Robust Tuning Approach for Two-Degree-of-Freedom PID Controllers," in European Control Conference (ECC09), August 23-26 2009, pp. 17881793, Budapest-Hungary.

[5] O. Arrieta, A. Visioli, and R. Vilanova, "PID autotuning for weighted servo/regulation control operation," Journal of Process Control, vol. 20, no. 4, pp. 472-480, 2010 .
[6] O. Arrieta and R. Vilanova, "Simple PID tuning rules with guaranteed $M_{s}$ robustness achievement," in 18th IFAC World Congress, August 28September 2, Milano-Italy, 2011.

[7] _ _ "Simple Servo/Regulation Proportional-Integral-Derivative (PID) Tuning Rules for Arbitrary $M_{s}$-Based Robustness Achievement," Industrial \& Engineering Chemistry Research, 2012, dOI: 10.1021/ie201655c.

[8] A. O' Dwyer, Handbook of PI and PID Controller Tuning Rules. McGraw-Hill, Londres, Inglaterra., 2006.

[9] V. Alfaro Ruiz, Model-reference robust tuning of 2DoF PI controllers for first- and second-order plus dead-time controlled processes. Journal of Process Control, p 358-374., 2012.

[10] V. Alfaro and R. Vilanova, Two-Degree-of-Freedom Proportional Integral Control of Inverse Response Second-Order Processes. $16^{\text {th }}$ Intenational Conference on System Theory, Control and Computing, 2012. 